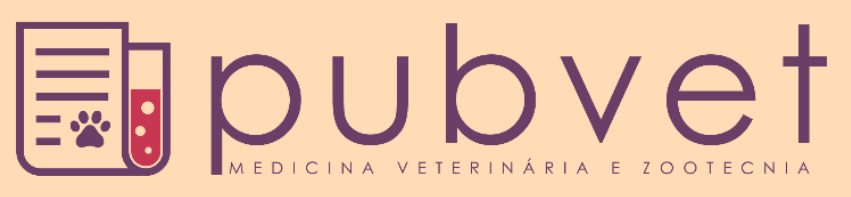

https://doi.org/10.31533/pubvet.v15n08a882.1-6

\title{
Efeito sedativo das diferentes doses de detomidina em equinos pelas vias intravenosa $\mathrm{e}$ intramuscular
}

\author{
Lucas Wamser Fonseca Gonzaga ${ }^{10}$, Emanuely Ramos Tameirão ${ }^{10}$, Luciana de Castro \\ Barcelos $^{10}$, Cintia Ferreira Antunes de Oliveira $^{10}$, João Vitor Fernandes Cotrim de Almeida ${ }^{\circ}$, \\ Paulo Fernandes Marcusso $^{30}$, Hugo Shisei Toma ${ }^{\circ} \bullet$ (D), Marcos Ferrante ${ }^{4 * \bullet}$ \\ ${ }^{1}$ Graduandos em medicina veterinária pela Universidade Federal de Lavras, departamento de medicina veterinária (UFLA), Lavras-MG, Brasil. \\ ${ }^{2}$ Doutorando do programa de pós-graduação em ciências veterinárias, UFLA, departamento de medicina veterinária, Lavras-MG, Brasil. \\ ${ }^{3}$ Docente do curso de medicina veterinária da Universidade Federal dos Vales do Jequitinhonha, Unaí-MG, Brasil \\ ${ }^{4}$ Docente do curso de medicina veterinária da Universidade Federal de Lavras, departamento de medicina veterinária, Lavras-MG, Brasil \\ *Autor para correspondência: marcos.ferrante@ufla.br
}

Resumo. O presente estudo teve por objetivo os efeitos sedativos das aplicações de detomidina, nos intervalos de doses de 50 a $80 \mathrm{ug} / \mathrm{kg}$ pela via intramuscular e de 20 a 40 $\mathrm{ug} / \mathrm{kg}$ pela via intravenosa. O trabalho foi baseado nos parâmetros farmacocinéticos de tempo máximo (Tmax), concentração máxima (Cmax) e tempo de meia vida $\left(\mathrm{T}^{1 / 2}\right)$. A relação entre a posição da cabeça e concentração plasmática foi estabelecida a partir de um modelo PK/PD. Se for tomado como base o tempo que o animal demora para retornar a cabeça para a posição de $100 \mathrm{~cm}$, é observado que este tempo é de 378, 396, 412 e 425 minutos para as doses de 50,60,70 e $80 \mathrm{ug} / \mathrm{ml}$ respectivamente, pela via intramuscular. É possível observar que o tempo de duração do efeito é de aproximadamente $78,101,122$, 138 e 150 minutos para as concentrações de 20, 25, 30, 35 e $40 \mathrm{ug} / \mathrm{kg}$ respectivamente, pela via intravenosa. O efeito considerado desejado durou 66, 88, 104, 127 e 139 minutos com as doses de $20,25,30,35$ e $40 \mathrm{ug} / \mathrm{kg}$ respectivamente, pela via intravenosa. O presente estudo concluiu que quanto maior a dose, maior será o tempo de duração do efeito independente da via. Pela via intramuscular ocorre um aumento de aproximadamente 20 minutos para cada $10 \mathrm{ug} / \mathrm{ml}$ de acréscimo na dose.

Palavras-chave: Alfa-2 agonistas, cavalos, modelamento pk/pd, sedação, simulação

\section{Sedative effect of different doses of detomidine in horses by intravenous and intramuscular routes}

Abstract. The present study aimed at the sedative effect of detomidine applications, in the dose range of 50 to $80 \mathrm{ug} / \mathrm{kg}$ intramuscularly, and from 20 to $40 \mathrm{ug} / \mathrm{kg}$ intravenously. The work was based on the pharmacokinetic parameters of maximum time (Tmax), maximum concentration (Cmax) and half-life ( $\mathrm{T} 1 / 2)$. The relationship between head position and plasma concentration was established using a PK/PD model. If the time taken for the animal to return the head to the $100 \mathrm{~cm}$ position is taken as a basis, it is observed that this time is $378,396,412$ and 425 minutes for the doses of 50, 60, 70 and $80 \mathrm{ug} / \mathrm{ml} \mathrm{respectively,} \mathrm{by} \mathrm{the} \mathrm{intramuscular} \mathrm{route.} \mathrm{It} \mathrm{is} \mathrm{possible} \mathrm{to} \mathrm{observe} \mathrm{that} \mathrm{the}$ duration of the effect is approximately $78,101,122,138$ and 150 minutes for the concentrations of $20,25,30,35$ and $40 \mathrm{ug} / \mathrm{kg}$, respectively, by the intravenous route. The effect considered desired lasted 66, 88, 104, 127 and 139 minutes with the doses of 20, $25,30,35$ and $40 \mathrm{ug} / \mathrm{Kg}$, respectively, through the intravenous route. The present study concluded that the higher the dose, the longer the duration of the effect, regardless of the 
route. Intramuscularly, there is an increase of approximately 20 minutes for each 10 $\mathrm{ug} / \mathrm{ml}$ increase in the dose.

Keywords: Alpha-2 agonists, horses, pk/pd modeling, sedation, simulation

\section{Efecto sedativo de las diferentes dosis de detomidina en equinos por via intravenosa e intramuscular}

Resumen. El presente estudio tiene como objetivo los efectos sedativos de las aplicaciones de la demotidina, en intervalos de dosis de 50 a $80 \mathrm{ug} / \mathrm{kg}$ por la vía intramuscular y de 20 a $40 \mathrm{ug} / \mathrm{kg}$ por la vía intravenosa. El trabajo tiene como base los parámetros fármaco cinéticos de tiempo máximo (Tmax), concentración máxima (Cmax) y tiempo de vida medio $\left(\mathrm{T}^{1 / 2}\right)$. La relación entre la posición de la cabeza y concentración plasmática fue establecida a partir de un modelo PK/PD. Si se toma como base, el tiempo que el animal tardo para retornar la cabeza para la posición de $100 \mathrm{~cm}$, es observado que este tiempo es de 378, 396, 412 y 425 minutos para las dosis de 50, 60, 70 y $80 \mathrm{ug} / \mathrm{mL}$ respectivamente por la vía intramuscular. Es posible observar que el tiempo de duración es de aproximadamente 78, 101, 102, 138 y 150 minutos para las concentraciones de 20, $25,30,35$ y $40 \mathrm{ug} / \mathrm{kg}$ respectivamente, por la vía intravenosa. El presente estudio concluyó que cuanto mayor es la dosis, mayor será el tiempo de duración del efecto independiente de la vía de aplicación. Por la vía intramuscular ocurre un aumento de aproximadamente 20 minutos para cada $10 \mathrm{ug} / \mathrm{ml}$ más en la dosis.

Palabras clave: Alfa-2 agonistas, caballos, modelaje pk/pd, sedativos, simulación

\section{Introdução}

Os sedativos alfa-2 agonistas são amplamente utilizados em medicina veterinária para as diferentes espécies, dentre elas a espécie equina (Tiburcio et al., 2014). Dentre os fármacos alfa-2 agonista, o cloridrato de detomidina é utilizado em equinos devido ao seu efeito analgésico visceral, e principalmente sedativo, ou ainda como uma medicação pré anestésica (Gaddini et al., 2018). O presente estudo simulou o efeito sedativo das doses de 50,60,70 e $80 \mathrm{ug} / \mathrm{kg}$ pela via intramuscular

Os conhecimentos em relação a farmacocinética e farmacodinâmica são de extrema importância para fazer predições de efeitos para diferentes doses de fármacos (Gaddini et al., 2018). Dessa forma, Mama et al. (2009) determinaram quais são as concentrações plasmáticas e seus respectivos efeitos sedativos a partir da doses de $30 \mathrm{ug} / \mathrm{kg}$ pelas vias intramuscular e intravenosa em quinos. Já Salonen et al. (1989) estabeleceram as concentrações plasmáticas da dose de $80 \mathrm{ug} / \mathrm{kg}$ utilizando as mesmas vias de administração; porém, não estabeleceram os efeitos sedativos para a mesma.

O objetivo deste estudo foi simular os efeitos sedativos das aplicações de detomidina, nos intervalos de doses de 50 a $80 \mathrm{ug} / \mathrm{kg}$ pela via intramuscular, e de 20 a $40 \mathrm{ug} / \mathrm{Kg}$ pela via intravenosa.

\section{Material e métodos}

Foi realizada uma simulação nas doses de 506070 e 80 ug/kg utilizando a via intramuscular e 20, $25,30,35$ e $40 \mathrm{ug} / \mathrm{kg}$ pela via intravenosa. O estudo foi baseado nos parâmetros farmacocinéticos definidos por Mama et al. (2009) como Tmax, Cmax e $\mathrm{T}^{1 / 2}$.

O Tmax corresponde ao tempo que o fármaco leva para atingir a Cmax. Para a via IM foi considerada a biodisponibilidade. O Tmax é um parâmetro constante para a via de administração escolhida, sendo atingido imediatamente após a administração.

No presente estudo, a concentração plasmática máxima foi calculada para cada dose utilizando a equação estabelecida por Toutain \& Bousquet-Mélou (2004), sendo, Cmax = Q/Vd. Onde "Q" representa a dose administrada e "Vd" o volume de distribuição. O Tmax para a via intramuscular foi estabelecido por Mama et al. (2009), assim como o $\mathrm{T}^{1 / 2}$. 
O decaimento do fármaco em relação ao tempo foi calculado a partir da concentração plasmática máxima para cada dose e via de administração e utilizando o $\mathrm{T}^{1 / 2}$.

A relação entre a posição da cabeça e concentração plasmática foi estabelecida a partir do modelo PK/PD de Gaddini et al. (2018). Foi estimado o efeito sedativo mediante o modelo PK/PD descrito por Gaddini et al. (2018), demonstrado na figura 1, que mediu o efeito sedativo a partir da posição da cabeça.

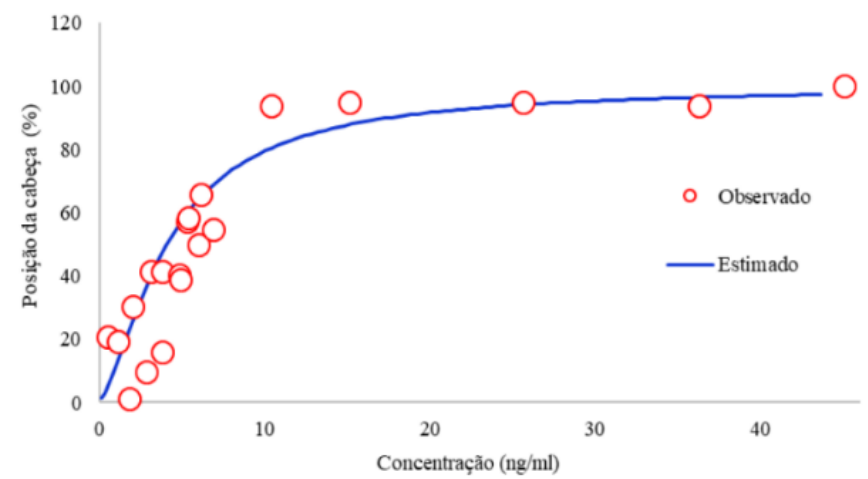

Figura 1. Modelo farmacocinético/farmacodinâmico de detomidina em equinos adaptado de (Gaddini et.al 2018)

\section{Resultados e discussão}

A figura 2 apresenta a variação da concentração plasmática com o passar do tempo, pela via intramuscular, demonstrando também a concentração plasmática mínima para se ter um efeito sedativo desejado.

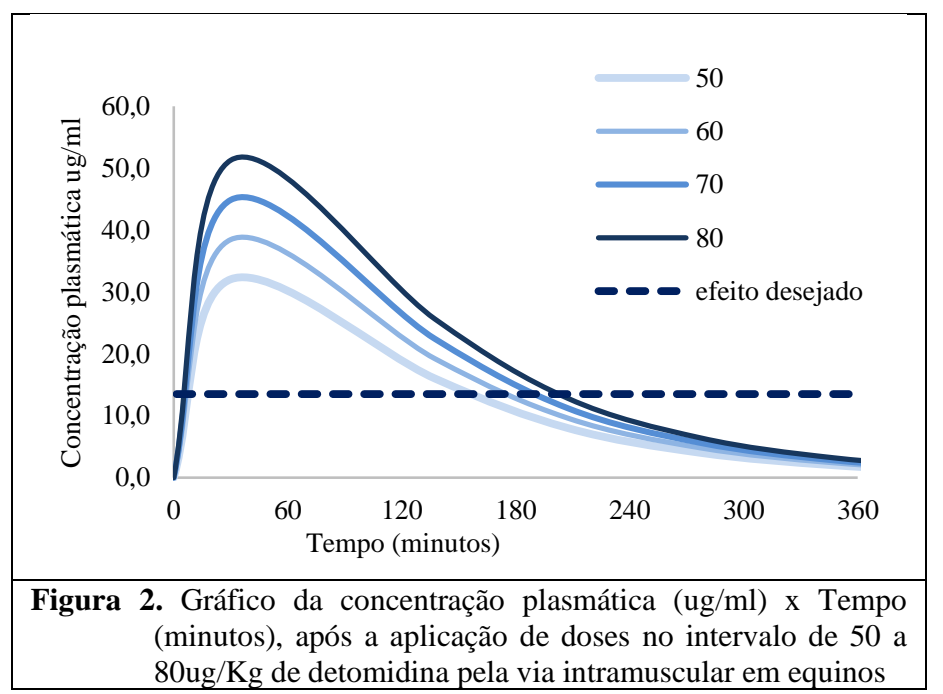

Pode-se notar que a concentração plasmática máxima se altera de acordo com a dose, porém o tempo que leva para a mesma ser atingida é o mesmo em todas as doses.

Também é possível se observar que tempo de duração do efeito desejado também aumenta de acordo com o aumento da dose. A duração do efeito foi de 150, 170, 190 e 210 minutos para as doses de $50,60,70,80 \mathrm{ug} / \mathrm{ml}$ respectivamente. Dessa forma, pode-se observar que o tempo de duração aumenta aproximadamente 20 minutos para cada $10 \mathrm{ug} / \mathrm{ml}$ de acréscimo na dose.

Na figura 3 é possível observar a variação da posição da cabeça em relação ao tempo. Por volta de 43 minutos após a aplicação do fármaco ocorre o efeito máximo, que será definido pela dose, podendo abaixar a cabeça até a altura de $36 \mathrm{~cm}$ no caso da dose de $80 \mathrm{ug} / \mathrm{kg}$, ou até $38 \mathrm{~cm}$ no caso da dose de $50 \mathrm{ug} / \mathrm{kg}$. À medida que o tempo passa o efeito também passa; porém, é possível observar que quanto maior a dose maior será o tempo de duração do efeito. Se for tomado como base o tempo que o animal 
demora para retornar a cabeça para a posição de $100 \mathrm{~cm}$, será observado que este tempo é de 378 , 396,412 e 425 minutos para as doses de 50, 60, 70 e $80 \mathrm{ug} / \mathrm{ml}$, respectivamente.

$\mathrm{Na}$ figura 4 é possível observar a variação da concentração plasmática no decorrer do tempo, com o fármaco aplicado pela via intravenosa.

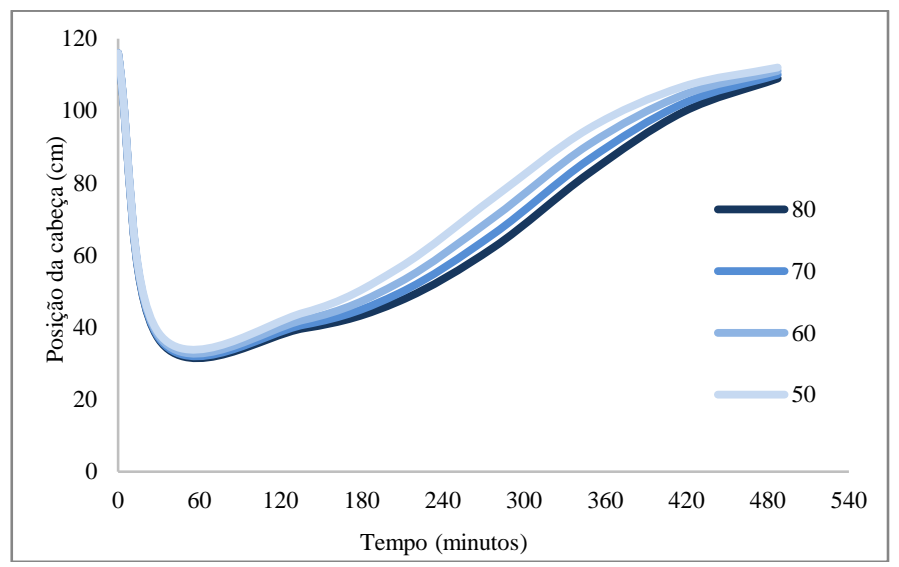

Figura 3. Relação entre a posição da cabeça (cm) X tempo (minutos), após a aplicação de detomidina pela via intramuscular em equinos

O fármaco atinge a concentração plasmática máxima imediatamente após a administração. É possível observar que o tempo de duração do efeito é de aproximadamente 78, 101, 122, 138 e 150 minutos para as concentrações de 20, 25, 30, 35 e $40 \mathrm{ug} / \mathrm{kg}$ respectivamente.

Na figura 5 é possível ver o que acontece com a posição da cabeça, a medida que o tempo passa quando o fármaco é aplicado pela via intravenosa.

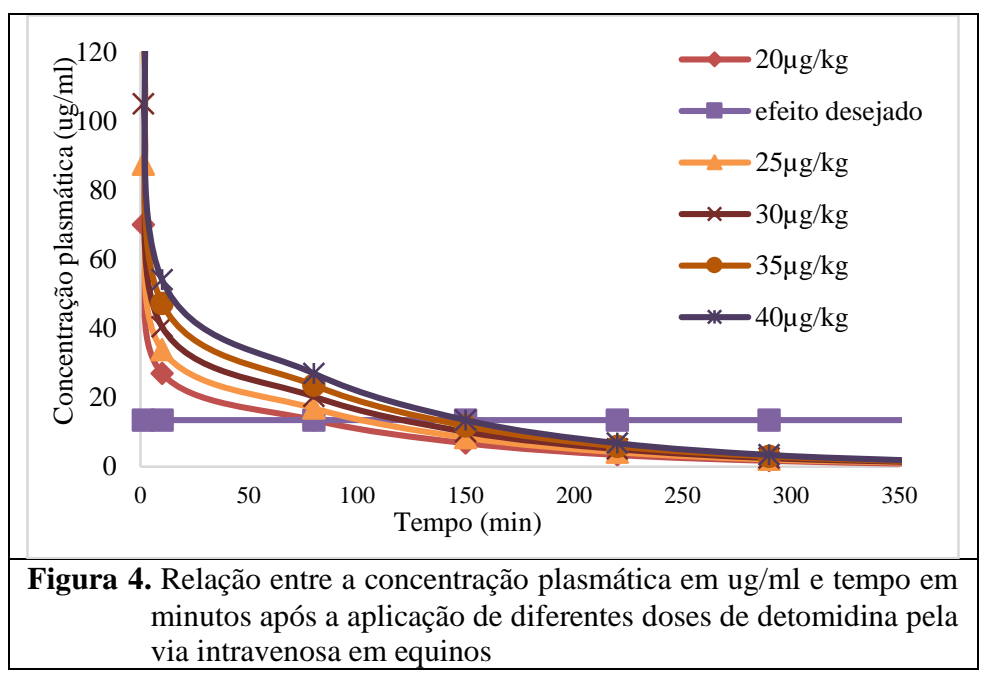

Dessa forma pode ser feita uma análise sobre o tempo que demora para a cabeça voltar da posição em que o efeito é considerado desejado, isto é, quando ela está a $45 \mathrm{~cm}$ do solo (Mama et al., 2009). O efeito considerado desejado durou 66, 88, 104, 127 e 139 minutos com as doses de 20, 25, 30, 35 e 40 $\mathrm{ug} / \mathrm{kg}$, respectivamente.

A concentração atingida após uma segunda administração em um intervalo menor as cinco meia vidas é superior que a concentração atingida na primeira dose o que pode determinar uma maior duração do efeito (Malfará et al., 2005). No presente estudo apresenta resultados da simulação após doses únicas portanto após a aplicação de doses repetidas a duração do efeito poderia ser maior.

Um aumento na dose determina um aumento da concentração plasmática máxima, e consequentemente, um aumento no período de eliminação do fármaco (Gaddini et al., 2018). Considerando que concentração necessária para o efeito desejado de detomidina é de $15 \mathrm{ug} / \mathrm{mL}$ 
(Gaddini et al., 2018), o presente estudo revelou o tempo de duração do efeito correspondente as mudanças nas doses e na via de administração.

Após a administração via intramuscular o fármaco necessita ser absorvido para ingressar na circulação sistêmica o que pode determinar maior tempo de disposição do fármaco no plasma quando comparado com a via intravenosa; porém, a detomidina rapidamente absorvida pela via intramuscular devido a sua alta lipofilicidade (Grimsrud et al., 2009). Isso poderia explicar os maiores tempos de duração do efeito observados entre as doses de 40 intravenoso (139 min) e 50 intramuscular (378 min), observados na presente simulação.

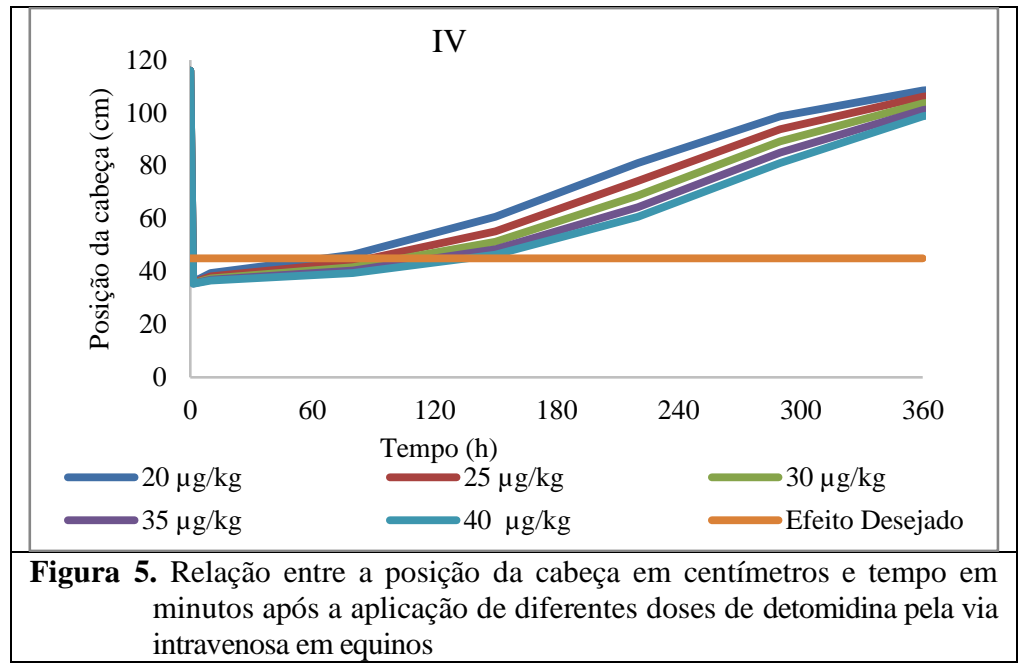

Segundo o estudo realizado por Mostafa et al. (1995), a dose de $20 \mathrm{ug} / \mathrm{kg}$ pela via intravenosa em asininos promoveu uma duração de 75 minutos no efeito. Segundo o nosso estudo a duração do efeito em equinos, utilizando a mesma dose, foi de 78 minutos o que indica que o comportamento nas espécies foi similar.

O presente estudo é de extrema importância pois serve como modelo de simulação para estudos futuros que avaliem a influência de outras fontes de variação do efeito, contribuindo para o bem-estar animal (Fontanela \& Ferrante, 2019). Além disso, é possível predizer o tempo de duração do efeito de cada dose, estabelecendo qual é a mais adequada segundo a necessidade do procedimento.

\section{Conclusão}

O presente estudo concluiu que quanto maior a dose, maior será o tempo de duração do efeito independente da via. Pela via intramuscular ocorre um aumento de aproximadamente 20 minutos para cada $10 \mathrm{ug} / \mathrm{ml}$ de acréscimo na dose.

\section{Referências}

Fontanela, M. A., \& Ferrante, M. (2019). Simulação do efeito antinociceptivo de detomidina em cavalos. Enciclopédia Biosfera, 16(29), 827-834. DOI: https://doi.org/10.18677/encibio_2019a66

Gaddini, L. V., Taffarel, M. O., \& Ferrante, M. (2018). Simulação do efeito sedativo de doses altas de detomidina em equinos. Pubvet, 12(11), 1-5. DOI: https://doi.org/10.31533/pubvet.v12n11a218.1-5

Grimsrud, K. N., Mama, K. R., Thomasy, S. M., \& Stanley, S. D. (2009). Pharmacokinetics of detomidine and its metabolites following intravenous and intramuscular administration in horses. Equine Veterinary Journal, 41(4), 361-365. DOI: https://doi.org/10.2746/042516409x370900

Malfará, W. R., Uyemura, S. A., \& Queiroz, R. H. C. (2005). Correlação entre dose/concentração plasmática e avaliação de alterações hepáticas e renais em ratos Wistar tratados com o esquema ROM. Revista Da Sociedade Brasileira de Medicina Tropical, 38(2), 167-172. DOI: https://doi.org/10.1590/s0037-86822005000200008 
Mama, K. R., Grimsrud, K., Snell, T., \& Stanley, S. (2009). Plasma concentrations, behavioural and physiological effects following intravenous and intramuscular detomidine in horses. Equine Veterinary Journal, 41(8), 772-777. DOI: https://doi.org/10.2746/042516409x421624

Mostafa, M. B., Farag, K. A., Zomor, E. L., \& Bashandy, M. M. (1995). The sedative and analgesic effects of detomidine (Domosedan) in donkeys. Journal of Veterinary Medicine Series A, 42(1-10), 351-356. DOI: https://doi.org/10.1111/j.1439-0442.1995.tb00387.x

Salonen, J. S., Vähä-Vahe, T., Vainio, O., \& VAKKURI, 0. (1989). Single-dose pharmacokinetics of detomidine in the horse and cow. Journal of Veterinary Pharmacology and Therapeutics, 12(1), 65-72. DOI: https://doi.org/10.1111/j.1365-2885.1989.tb00643.x

Tiburcio, M., Oliveira, M. S., Martini, M. V., Dias, L. G. G. G., \& Mattos Junior, E. (2014). Acepromazina, detomidina ou xilazina na sedação em equinos: efeitos hematológicos e bioquímicos. Revista Acadêmica Ciência Animal, 12(1), 35-44. DOI: https://doi.org/10.7213/academica.12.01.ao04

Toutain, P.-L., \& Bousquet-Mélou, A. (2004). Volumes of distribution. Journal of Veterinary Pharmacology and Therapeutics, 27(6), 441-453. DOI: https://doi.org/10.1111/j.1365$\underline{2885.2004 .00602 . x}$

Histórico do artigo:

Recebido: 5 de março de 2021

Aprovado: 24 de abril de 2021.
Licenciamento: Este artigo é publicado na modalidade Acesso Aberto sob a licença Creative Commons Atribuição 4.0 (CC-BY 4.0), a qual permite uso irrestrito, distribuição, reprodução em qualquer meio, desde que $\mathrm{o}$ autor $\mathrm{e}$ a fonte sejam devidamente creditados. 